\title{
Study of Evacuation Techniques in the Event of a Night Fire at a Dementia Group Home - Method of Transferring Evacuees from Their Beds to the Floor
}

\author{
Tomoya Miyasaka, Mai Matsumoto, Mamiko Kamoshida, Keiko Kawashima, Nobuhito Ohtsu, \\ Takahiro Tsukame, Nobuyuki Abe, Hiroyuki Tamura
}

\begin{abstract}
Assuming a nighttime situation in a dementia group home, assistance operations were performed under the condition of 1 assistant, transfer from a supine position to the floor with full assistance, and a low bed (height $=0.4 \mathrm{~m}$ ). Assistance operations were performed under combinations of 4 conditions: starting assistance from the head side, starting assistance from the foot side, female assistants with male evacuees, and male assistants with female evacuees. The assistance time was analyzed by dividing it into three operation phases, and it was found that the operation time of female assistants was longer. Further, when assistance was started from the head side, it took longer to pull the evacuee to the front of the bed, whereas when assistance was started from the foot side, it took longer to lower the evacuee from the bed to the floor. Since the purpose of the assistance operation was to evacuate evacuees from a fire, the experimental results and techniques of shortening the assistance time were examined. Considering that assistance may be given by people who do not have much strength, we showed the possibility of utilizing tools to reduce friction during the operation, such as slide sheets, to help pull an evacuee on the bed, to the front of the bed. As regards the assistance operation of lowering an evacuee from the bed to the floor, a technique was suggested of holding the evacuee's feet, pulling the buttocks to the front of the bed, and after placing the feet on the floor, lowering the evacuee onto the floor from the buttocks, followed by the waist, back, head and neck.
\end{abstract}

Index Terms - evacuation technique, people unable to self-evacuate, dementia group home

\section{INTRODUCTION}

In Japan, one of the welfare policies for the elderly is the Nursing Care Insurance System [1]. Under this care insurance system, welfare services can be received upon application by

This work was supported by JSPS KAKENHI Grant Number JP 17K04232.

Tomoya Miyasaka, Department of Physical Therapy, Faculty of Health Sciences, Hokkaido University of Science, Sapporo, Hokkaido, Japan.

Mai Matsumoto, Faculty of Rehabilitation, Sapporo Miyanosawa Neurosurgery Hospital, Sapporo, Hokkaido, Japan.

Mamiko Kamoshida, Department of Physical Therapy, Faculty of Health Sciences, Hokkaido University of Science, Sapporo, Hokkaido, Japan.

Keiko Kawashima, Department of Physical Therapy, Faculty of Health Sciences, Hokkaido University of Science, Sapporo, Hokkaido, Japan.

Nobuhito Ohtsu, Research and Development Div., National Research Institute of Fire and Disaster, Chofu, Tokyo, Japan.

Takahiro Tsukame, Fire and Disaster Investigation Div., National Research Institute of Fire and Disaster, Chofu, Tokyo, Japan.

Nobuyuki Abe, Research and Development Div., National Research Institute of Fire and Disaster, Chofu, Tokyo, Japan.

Hiroyuki Tamura, Research and Development Div., National Research Institute of Fire and Disaster, Chofu, Tokyo, Japan. elderly persons or their families. Welfare services include a home-based type, facility-based type and community-based type. Some welfare services of facility- and community-based types are offered to elderly people who cannot support themselves independently at home so that they can enter a facility and receive care in daily life [2]. Facilities for the elderly include special old people's nursing homes, large-scale institutions such as elderly health care facilities, and small-scale facilities such as dementia group homes [3]. All facilities are required by the Fire Service Law [4] to have fire evacuation drills at least twice a year, and these are conducted by each facility.

Since residents are elderly and some have dementia, it is difficult for many of them to evacuate on their own, and evacuation is therefore aided by assistants [5]. Methods of assisted evacuation are indicated in a legally mandated instruction manual [6] with a suitable technique being implemented by the parties involved depending on the situation. In particular, a dementia group home, which is a small-scale facility, should have a system of at least one night shift worker for nine people with dementia [7]. By law, sprinkler installation is obligatory regardless of the size of floor space even if it is a small facility [8], but if a fire occurs at night, depending on the situation, one assistant is needed to evacuate each resident quickly to a safe place. We are therefore conducting research on evacuation techniques for residents who have difficulty in self-evacuation in the event of a night fire at a facility for the elderly [9]-[13].

This study looked at evacuation techniques in the event of a night fire at a dementia group home. Specifically, assistance experiments were carried out under the condition of 1 assistant, transfer from bed to floor with full assistance, and a low bed (height $=0.4 \mathrm{~m}$ ). Based on the results, techniques of rendering assistance to lower the evacuee (resident) from the bed to the floor, which is the first step in evacuation, were examined.

\section{METHOD}

\section{A. Subjects and experimental methods}

The subjects were healthy volunteers, and played the roles of assistants and evacuees. The assistants attempted to lower evacuees who were lying supine on a bed to the floor, and this experimental operation was recorded with a video camera.

The subjects included 3 healthy men and 3 healthy women aged 20 years old or more. The 3 men had a height of $167.7 \pm 4.6 \mathrm{~cm}$, weight of $61.3 \pm 3.5 \mathrm{~kg}$, and grip strength of (right) $44.7 \pm 6.4 \mathrm{~kg}$, (left) $46.2 \pm 4.9 \mathrm{~kg}$, whereas the 3 women 


\section{Study of Evacuation Techniques in the Event of a Night Fire at a Dementia Group Home - Method of Transferring Evacuees from Their Beds to the Floor}

had a height of $158.2 \pm 2.0 \mathrm{~cm}$, weight of $46.0 \pm 3.6 \mathrm{~kg}$, and grip strength of (right) $29.0 \pm 1.0 \mathrm{~kg}$, (left) $25.8 \pm 0.3 \mathrm{~kg}$. All subjects were students who studied physical therapy at university, and had taken three 6-week clinical training instruction courses on how to give assistance. Their grip strength was measured by a Smedley-type dynamometer [14]. The measurement of grip strength was taken while standing, performed twice alternately for the left and right hands, and the maximum values of right and left were used as data.

The assistance operation was recorded with a video camera (30 fps) from two directions, obliquely upward and sideway. The phases (operation steps) of the assistance were determined from the video file obtained, and their time (s) was determined.

\section{B. Transfer from bed to floor}

Assuming a typical room configuration at a dementia group home, the assistant lowered the evacuee from the bed $0.4 \mathrm{~m}$ above the floor, to the floor just in front the bed. There were two techniques for lowering the evacuee from the bed. In one technique, "Assistance Technique 1" (Fig. 1), the assistant told the envisaged evacuee to evacuate, and immediately started to give help by lowering the evacuee's head on the floor, and then the whole body. In the other technique, "Assistance Technique 2" (Fig. 2), after the assistant told the envisaged evacuee to evacuate, assistance was started by pulling the evacuee to the front of the bed, lowering the feet to the ground, and then the whole body. The assistant repeated the two techniques three times each. Subsequently, assistants and evacuees interchanged roles. A total of 180 operations were performed.

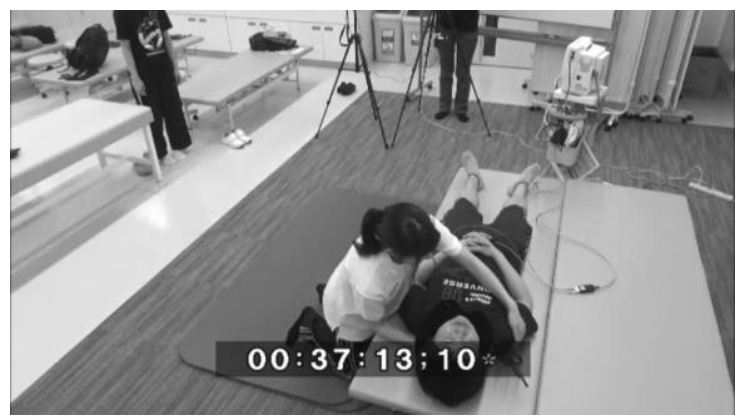

Fig. 1 Assistance Technique 1

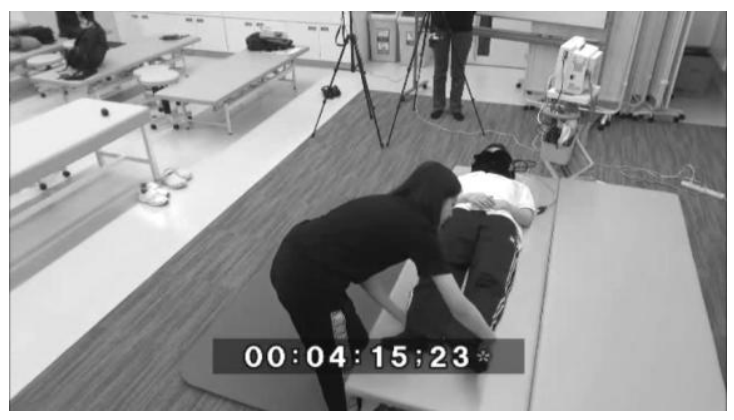

Fig. 2 Assistance Technique 2

\section{Research ethics and safety considerations}

This study was undertaken after giving an explanation to subjects in advance, and obtaining their consent based on Hokkaido University of Science Research Ethics Application No. 224. Regarding safety considerations in the experiment, two soft mats were laid on the floor to prevent trauma even if the evacuee fell from the bed. In addition, a collar protecting the neck was attached to the evacuee, and the electrocardiogram waveform, heart rate, and percutaneous arterial blood oxygen saturation $\left(\mathrm{SpO}_{2}\right)$ were monitored. Personal information protection was taken into account when shooting videos, so that individuals cannot be identified.

\section{REsults}

The subjects performed assistance operations, and the operations were recorded with a video camera. There were no abnormalities in values of the electrocardiogram, heart rate, and $\mathrm{SpO}_{2}$ of the evacuee throughout the experiment. The file recorded by the video camera was replayed at intervals of 30 fps using a personal computer and video editing software to determine the phase of the assistance operation. In both Assistance Technique 1 and Assistance Technique 2, three phases were set. The first phase was from when the assistant touched the evacuee's body until the evacuee started to move (Figs. 3, 6), the second phase was until a part of the evacuee's body came in contact with the floor (Figs. 4, 7), and the third phase was when the body of the evacuee was lowered to the floor, and the area of the contact surface between the evacuee's back and the floor was a maximum (Figs. 5, 8). The operation time of each phase was aggregated.

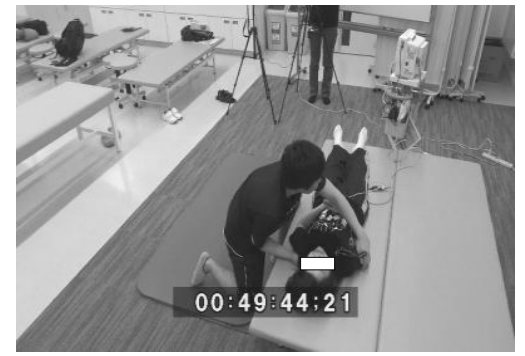

Fig. 3 First phase (Assistance Technique 1)

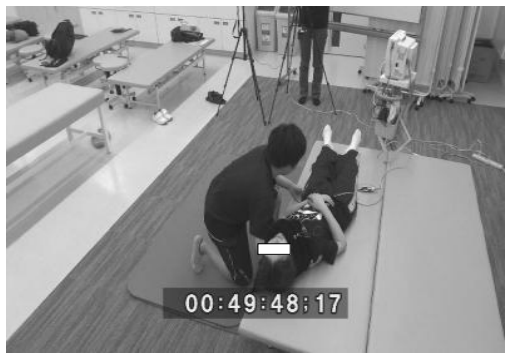

Fig. 4 Second phase (Assistance Technique 1)

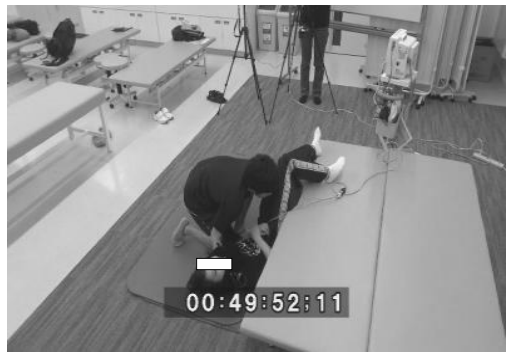

Fig. 5 Third phase (Assistance Technique 1) 


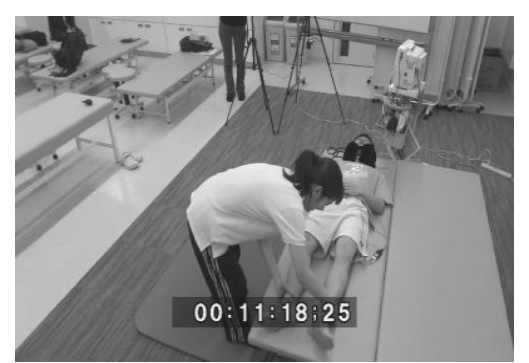

Fig. 6 First phase (Assistance Technique 2)

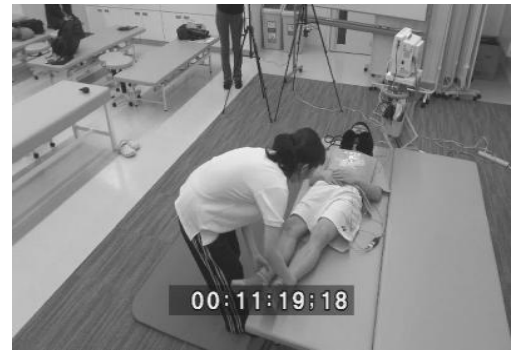

Fig. 7 Second phase (Assistance Technique 2)

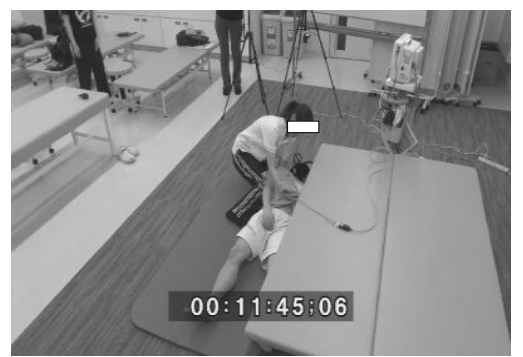

Fig. 8 Third phase (Assistance Technique 2)

\section{DISCUSSIONS}

\section{A. Assistance time}

Focusing on the assistant's force used in the operation, combinations of four conditions were compared (Table 1): a female assistant with a male evacuee, a male assistant with a female evacuee, and two types of assistance techniques. The

Table 1 Four conditions

$\begin{aligned} & \text { Condition } 1 \begin{array}{l}\text { Female assistant/Male evacuee } \\ \text { Assistance Technique } 1\end{array} \\ & \text { Condition } 2 \begin{array}{l}\text { Female assistant / Male evacuee } \\ \text { Assistance Technique } 2\end{array} \\ & \text { Condition 3 } \begin{array}{l}\text { Male assistant / Female evacuee } \\ \text { Assistance Technique 1 }\end{array} \\ & \text { Condition 4 } \begin{array}{l}\text { Male assistant / Female evacuee } \\ \text { Assistance Technique } 2\end{array} \\ & \text { For all conditions } \mathrm{N}=27 \text { (including repetitions) }\end{aligned}$

assistance operation for each condition and the average time for each phase are shown in Fig. 9. Female assistants took longer than male assistants for both assistance techniques. In addition, as regards the average time for each phase, the second phase of Assistance Technique 1 and the third phase of Assistance Technique 2 accounted for half or more of the total time, respectively.

Two-way configuration analysis of variance with repetition was used for the null hypothesis where there was no difference of assistance time due to assistant gender or assistance technique. Similarly, a parametric multiple comparison test (Tukey-Kramer technique) was used for the null hypothesis that there was no difference in the time of each phase. Both were performed with a risk rate of $5 \%$ to conduct statistical analysis.

Combinations between which there was a significant difference of average assistance time were Condition 1 and Condition 3, Condition 1 and Condition 4, Condition 2 and Condition 3. In particular, when Conditions 1 and 3 were compared, the assistance time of Condition 1 was significantly longer than that of Condition 3 by 18.93 seconds, and the second phase was 14.77 seconds longer. The reason why the assistance time of Condition 1 was longer, was examined. The average values of left- and right-hand grip, which are a basic attribute of the subjects, were 27.4 \pm 1.9 $\mathrm{kg}$ for women and $45.4 \pm 5.2 \mathrm{~kg}$ for men. Grip strength is correlated with the muscle strength of skeletal muscle groups [15], hence it may be concluded that the force exerted by a female assistant is weaker than that of a male assistant. Further, the average values of body weight, which are a basic attribute of the subject, were $46.0 \pm 3.6 \mathrm{~kg}$ for women and $61.3 \pm 3.5 \mathrm{~kg}$ for men. From the above, a female assistant had less force to assist an evacuee than a male assistant, and in addition, a female assistant had to lower a male evacuee who is heavier than a female evacuee. It is thought that this was why the assistance time was longer. In facilities such as dementia group homes in Japan, there are many female staff [16], therefore the conditions with longer assistance time in this study mirror the actual situation.

\section{B. Assistance technique}

Differences of assistants and operation phases were examined for Assistance Technique 1. For Conditions 1 and 3 of Fig. 9 in which there was a significant difference of average assistance time, in particular, the second phase of Condition 1 took about 15 seconds longer than the second phase of Condition 3. Examining the second phase of Assistance Technique 1 by means of videos revealed that it took time for the assistant to pull the evacuee, who was lying supine on the bed, to the front of the bed. The assistant was pulling the evacuee's buttocks and trunk alternately to the front. In the case of a female assistant, the distance pulled to the front in one assistance motion was short, so the operation had to be repeated many times. This is considered to be due to the assistant's weak force.

Differences between assistants and operation phases were also examined for Assistance Technique 2. There was no significant difference between average assistance times for Condition 2 and Condition 4 in Fig. 9. The third phase of Condition 2 took about 12 seconds longer than the third phase of Condition 4. When the third phase of Assistance Technique 2 was examined by videos, it apparently took longer to lower the evacuee, who was at the front of the bed, onto the floor. Whichever the assistant, if the evacuee were rapidly lowered from the front of the bed to the floor, an impact could have 


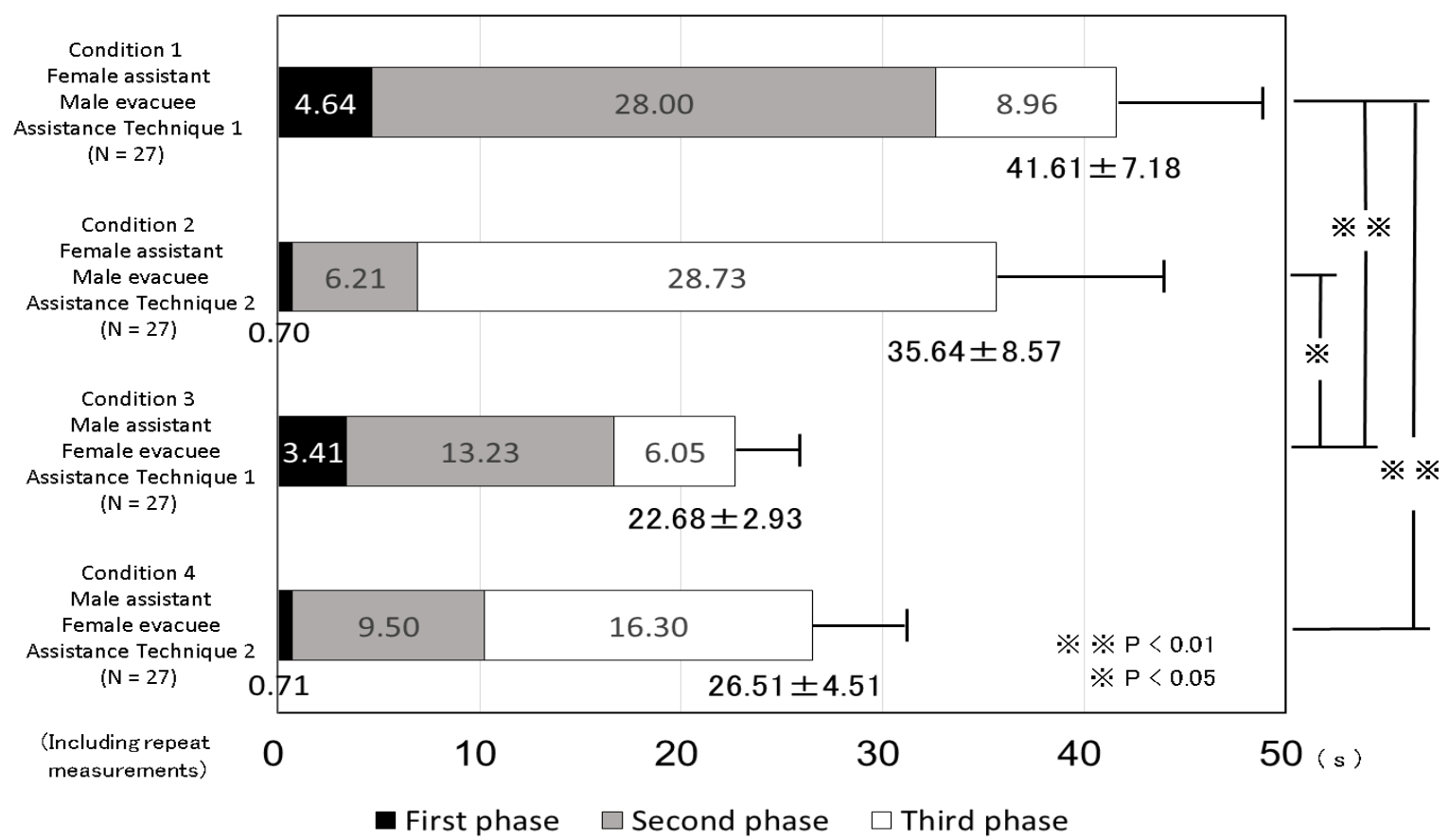

Fig.9 Assistance phases and times

occurred, which was similarly caused by falling. Therefore, while carefully protecting the head, the evacuee was lowered slowly in the order of buttocks, waist, back, shoulders and head. As in the second phase of Assistance Technique 1, the body parts that could be moved by one operation were limited locally to the buttocks and lower legs, and the operation had to be repeated many times.

Comparing Assistance Technique 1 and Assistance Technique 2, there was no significant difference in the average assistance time for Conditions 1 and 2 between female assistants, and Conditions 3 and 4 between male assistants. From this, it may be concluded that there is no significant difference in assistance time between the two techniques in the case of persons having the same level of forces used in assistance operation.

There was a significant difference between Condition 1 and Condition 3 when a female assistant was compared with a male assistant. From this, it can be concluded that the assistant's force strongly influences the time of the operation in Assistance Technique 1. In particular, when the second phase was short, the whole assistance time was short. In other words, in the second phase, if the evacuee can be quickly pulled by a weak force, an assistant with weak force may be able to assist in a short time. For Assistance Technique 2, there was no significant difference in assistance time depending on the evacuee. From this, it can be concluded that the influence of the assistant's strength in Assistance Technique 2 is not as great as for Assistance Technique 1. Thus, there can be evidently room for shortening the assistance time by improving the assistance procedure.

\section{Shortening the assistance time}

Since the purpose of the assistance operation was to evacuate evacuees from a fire, the results were used to examine techniques of shortening the operation. In particular, it was considered that an assistance operation might have to be performed by persons with weaker force such as the female assistants who carried out the experiments. Particular attention was also paid to the second phase of Assistance Technique 1 which included an operation for pulling the evacuee to the front of the bed, and the second phase and third phase of Assistance Technique 2 which included an operation for pulling the evacuee to the front of the bed and lowering him or her onto the floor.

According to the experimental results, in the second phase of Assistance Technique 1, the assistant pulled each part of the evacuee's body again and again. It was therefore considered how the assistant could pull the evacuee to the front end of the bed with fewer actions. In the caregiving field, a low friction slide sheet [17], which can help even a weaker assistant transfer a person sleeping on a bed, may be used.

In terms of the muscle activity of each part of the body, when the person is horizontally moved on the bed, comparison between using human hands and using a slide sheet reveals that the required strength of the muscle activity is about 30 to $70 \%$ less when a slide sheet is used [18]. This suggests the possibility that fewer assistance operations may be required and the evacuee can be pulled in a short time even by a weak force by using a tool for reducing friction such as a slide sheet (Fig. 10). When using a slide sheet, it should be considered whether the slide sheet should be laid in advance between the bed and the sheets, or the sheet should be kept in another place at normal times, and if the assistant brings a slide sheet in, it should not be forgotten that there is the extra work of laying the slide sheet between the bed and the evacuee before performing the assistance operation.

We examined the second phase and third phase of Assistance Technique 2 to make them more efficient without using auxiliary tools. According to the lever principle based on biomechanics [19], an assistance operation to lift part of the body of an evacuee lying supine on the bed corresponds to 
a Type 2 lever operation [20], and it may then be easier to

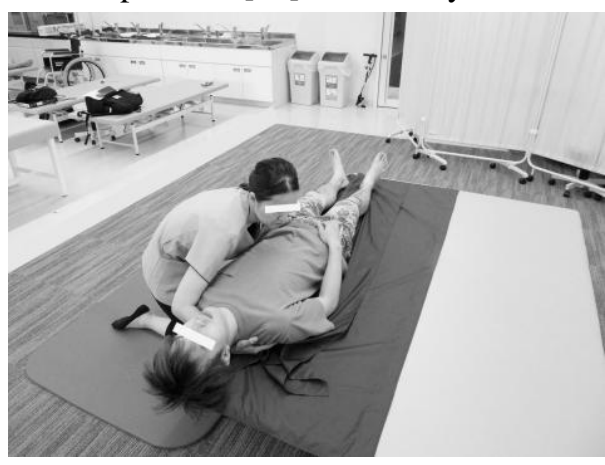

Fig.10 Using a slide sheet

move both feet than the head. Here, the assistant holds the evacuee's feet, pulls them until the evacuee's buttocks come to the front of the bed, and then lowers both soles on the floor (Fig. 11). Next, an operation that can be considered is that the assistant lowers the evacuee onto the floor in the order of buttocks, waist, back, head and neck while supporting both the lower limbs and back of the evacuee with both hands (Fig. 12). In the second phase, the evacuee is carefully pulled sufficiently close to the front of the bed so that the evacuee does not fall down, and in the third phase, not to give a large impact when lowering the buttocks on the floor. Subsequently, care should also be taken not to give a shock or allow excessive joint movement when lowering the neck.

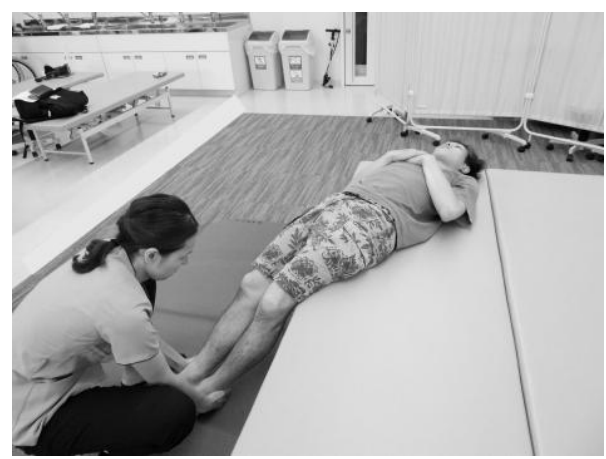

Fig.11 Assistance Technique 2, Second phase

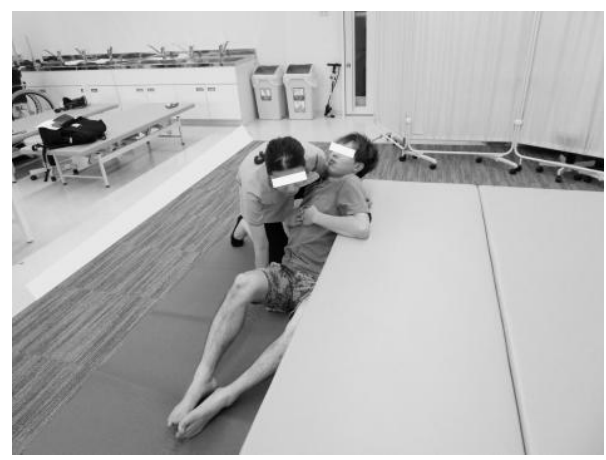

Fig.12 Assistance Technique 2, Third phase

\section{Limitations of the study}

The subjects of this study were healthy volunteers. In the experiment, a treatment bed was used, and bedding such as sheets and mats which increase friction were not used. There was no furniture in the area, which is different from an actual living room environment. Further, a night fire was assumed, nevertheless, under a bright environment with lighting, there was not smoke or a wet floor surface with water discharged by a sprinkler.

\section{SUMMARY}

Assuming a nighttime situation in a dementia group home, assistance operations were performed under the condition of 1 assistant, transfer from a supine position to the floor with full assistance, and a low bed (height $=0.4 \mathrm{~m}$ ). Assistance operations were performed under combinations of 4 conditions: starting assistance from the head side, starting assistance from the foot side, female assistants with male evacuees, and male assistants with female evacuees. The assistance time was analyzed by dividing it into three operation phases, and it was found that the operation time of female assistants was longer. Further, when assistance was started from the head side, it took longer to pull the evacuee to the front of the bed, whereas when assistance was started from the foot side, it took longer to lower the evacuee from the bed to the floor.

Since the purpose of the assistance operation was to evacuate evacuees from a fire, the experimental results and techniques of shortening the assistance time were examined. Considering that assistance may be given by people who do not have much strength, we showed the possibility of utilizing tools to reduce friction during the operation, such as slide sheets, to help pull an evacuee on the bed, to the front of the bed. As regards the assistance operation of lowering an evacuee from the bed to the floor, a technique was suggested of holding the evacuee's feet, pulling the buttocks to the front of the bed, and after placing the feet on the floor, lowering the evacuee onto the floor from the buttocks, followed by the waist, back, head and neck.

In future, other techniques of giving assistance using a slide sheet and assistance for lowering from the bed to the floor will be considered. Additionally, an assistance technique for evacuating an evacuee in the horizontal direction on the floor will also be considered. In order to shorten the assistance operation time and reduce the assistance burden, evacuation techniques that can be implemented by weaker persons will continue to be evaluated.

\section{REFERENCES}

[1] Yasunori Makino, Miyo Araki, "Aiming at sustainable elderly care system $\sim$ Long-term care insurance system and turning point in $2025 \sim$, Journal of the Kobe University of Welfare, vol.19 (1), 2018, pp.119-143.

[2] Hideaki Sato, "The Current State and Issues of Supplying Nursing Home Services under the Public Long-term Care Insurance System," Bull of Obihiro Otani Junior College, vol.51, 2014, pp.47-56.

[3] Haruyasu Yamaguchi, Kunihiko Hayashi, Takao Ando, Ken-ichi Inoue,Kaoru Sasaki, Kimiko Sekimoto, Masahiko Shigezawa, Takahisa Hayashida,Naoto Miyazaki, Kazuyoshi Furukawa, Akiko Konno, Takanobu Hosaka,Katsumi Maeda, "Study Committee of the Effect of Group Home Care on Dementia Study of the effect of group home care in group home for people with dementia," Tokyo Journal of Dementia Care Research, vol.2, 2018, pp.103-115.

[4] Yukiko Inoue, Satoshi Ishii, Tatsuya Nishino, “ ACTUAL CONDITIONS AND PROBLEMS OF FIRE SAFETY IN THE GROUP LIVING FOR THE ELDERLY WITH DEMENTIA AND THE COMMUNITY BASED MULTI-CARE FACILITY," AIJ $J$. Technol. Des., vol.18(40), 2012, pp.1023-1028.

[5] Masami Shinozaki, Ai Sekizawa, Kyoichi Kobayashi, Katsunori Sasaki, “ International Comparative Analysis on Regulatory 
Provisions and Codes of Fire Evacuation Safety for Physically Challenged People," Bulletin of Japan Association for Fire Science and Engineering, vol.66(2), 2016, pp.21-30.

[6] International Fire Service Information Center, Fire Prevention $\begin{array}{llrl}\text { Administration in } 2013 . & \text { pp.80, }\end{array}$ http://www.kaigai-shobo.jp/pdf/Fire_prevention_administrative_jpn.p df

[7] Yoko Furukawa, Akiko Takahashi, Yuji Hasemi, “A STUDY FOR THE FIRE SAFETY PLANNING OF GROUP HOMES FOR THE AGED WITH SENILE DEMENTIA,” J.Environ.Eng., AIJ, No.613, 2007, pp.9-14

[8] International Fire Service Information Center, Cabinet Order for Enforcement of the Fire Service Act, 2015, pp.88-89, http://www.kaigai-shobo.jp/pdf/Cabinet_Order_for_Enforcement.pdf

[9] Tomoya Miyasaka, Yuji Tanaka, Keiko Kawashima, Yoshimi Tanahashi, Shiho Namba, Takahiro Tsukame, Hiroyuki Tamura, Nobuyuki Abe, Ken'ichi Takanashi, Sanae Matsushima, Daisuke Kozeki, Takayuki Saito, Ryo Tazawa, Jun Ito, Noriyuki Miyashita, "A Study on Evacuation Capability of Residents in Elderly Facilities," Proceedings of JAFSE Annual Symposium 2015, 2015, pp.44-47.

[10] Tomoya Miyasaka, Takahiro Tsukame, Hiroyuki Tamura, Nobuyuki Abe, Ken'ichi Takanashi, Kosuke Fujii, Daisuke Kozeki, Takayuki Saitou, Masanobu Yamazaki, Jun Ito and Kohei Takatsuka, “ Consideration on Preparation for Evacuation with Assistance and its Required Time at Fire Drill of a Group Home for Dementia Patients," Proceedings of JAFSE Annual Symposium 2016, 2016, pp.82-83.

[11] Tomoya Miyasaka, Mai Matsumoto, Keiko Kawashima, Mamiko Kamoshida, Takumi Ino, Takahiro Tsukame, Hiroyuki Tamura, Nobuyuki Abe, Kosuke Fujii, and Daisuke Kozeki, "Study on Dragging Evacuation Method at Fire Drill of a Group Home for Dementia Patients," Proceedings of JAFSE Annual Symposium 2017, 2017, pp.152-153.

[12] Tomoya Miyasaka, Keiko Kawashima, Mamiko Kamoshida, Katsuyuki Toide, Noriyuki Sugawara, Shinya Hashimoto, Atsushi Matoba, Kouhei Takatsuka, Kenji Edazawa and Masanori Kawai, "A development of evacuation vehicles for stairs that can descend while getting on wheelchair," Proceedings of JAFSE Annual Symposium 2018, 2018, pp.152-153.

[13] Tomoya Miyasaka, Mina Tanaka, Keiko Kawashima, Mamiko Kamoshida, Takahiro Tsukame, Hiroyuki Tamura, Nobuhito Ohtsu, Nobuyuki Abe and Kosuke Fujii, "Study of means of evacuation at elderly facilities on fire; maximum pull force of transfer with assistance for the rescuee on the bed," Proceedings of JAFSE Annual Symposium 2019, 2019, pp.308-309.

[14] Katsushi YOSHIDA, Yasuo YOSHIHUKU, Yoshinori ADACHI, Takashi AOKI, "An Examination into the Dependence of the Grip Strength Measured with a Popular Type (Smedley type ) Dynamometer on the Grip Width \& Experience, and its Circadian Change,” Journal of Mind-Body Science, vol.6(1),1997, pp.17-25.

[15] Laurentani F, Russo C, Bandinelli S, et al., "Age-associated changes in skeletal muscles and their effect on mobility: an operational diagnosis of sarcopenia," J Appl Physiol, vol.95, 2003, pp.1851-1860.

[16] Mika Kunisada, "RESEARCH IN RELATION WITH THE CARE WORKING HOUR OF LONG TERM CARE WORKS IN WELFARE INSTITUTE FOR ELEDERLX THEIR LEVEL OF BURDEN AND LEVEL OF ACHIEVEMENT," Japanese Journal of Human Sciences of Health-Social Services, vol.17(1), 2011, pp.1-8.

[17] Larson RE, Murtagh EM, Rice MS., "Forces involved when sliding a patient up in bed," Work, vol.59(3), 2018, pp.439-448

[18] Youko Shiraishi, Satomi Suzuki, "Comparison of muscle activity in each body part by a method in which a moving a horizontal person side-to-side on a bed is performed by a manual handling or using a slide seat," JOURNAL OF MIE PREFECTURAL COLLEGE OF NURSING, vol.20, 2016, pp.63-68.

[19] David A. Winter,'BIOMECHANICS and MOTOR CONTROL of HUMAN MOVEMENT FOURTH EDITION," WILEY JOHN WILEY \& SONS, INC, 2009, pp.1-13.

[20] Koichi Ogawa, Kyoko Kitamura, "Body Mechanics for Careworkers," Tokyo Denki University Press, 2016, pp.66. 